\title{
A functional explanation for the effects of visual exposure on preference
}

\author{
Mark A Changizi \\ Sloan - Swartz Center for Theoretical Neurobiology, MC 139-74, California Institute of Technology, \\ Pasadena, CA 91125, USA; and Department of Cognitive Science, Rensselaer Polytechnic Institute, \\ Troy, NY 12180-3590, USA—address for correspondence; e-mail: changizi@rpi.edu
}

\section{Shinsuke Shimojo}

Division of Biology, Computation and Neural Systems, MC 139-74, California Institute of Technology, Pasadena, CA 91125, USA; and JST ERATO Shimojo Implicit Brain Function Project;

e-mail: sshimojo@its.caltech.edu

Received 15 February 2007, in revised form 10 March 2008; published online 2 October 2008

\begin{abstract}
Visual exposure to an object can modulate an observer's degree of preference for it, initially enhancing preference (a 'familiarity preference' regime), and eventually lowering it again (a 'novelty preference' regime). Here we investigate whether there may be a functional advantage to modulating preference in this way. We put forth the simple hypothesis that degree of preference for an object of type $\mathrm{X}$ is the brain's estimate of the expected utility of acting to obtain X. In the light of this view of what preferences fundamentally represent, we are able to explain the 'exposure effect' and many of the connected phenomena.
\end{abstract}

\section{Introduction}

Seeing an object can modulate one's degree of preference for it, in some cases enhancing degree of preference, and in other cases lowering it. At first glance, such 'exposure effects' (Zajonc 1968; Zajonc et al 1972; Bornstein 1989; Shimojo et al 2003) seem utterly irrational. After all, the value of an object in the world does not in most cases change upon looking at it! Although there are a number of hypothesized potential mechanisms underlying these effects (see Eagly and Chaiken 1993), such as response competition (Harrison 1968; Harrison and Zajonc 1970), positive habituation and boredom (Berlyne 1970), and perceptual fluency (Bornstein and D’Agostino 1992; Reber et al 1998), little attention has been given to possible functional advantages of these enigmatic effects. Preferences fundamentally guide our goal-directed actions, and it would be surprising if evolution had let our preferences be capriciously affected by visual exposure. Here we investigate the idea that the effects of visual exposure on preference in fact tend to guide an observer toward optimal goal-directed behavior.

In particular, our basic assumption is simply that the degree of preference one has for some object $\mathrm{X}$ is an indicator of the subjective expected utility of engaging in goal-directed behavior toward object X. Or, more informally, our fundamental hypothesis is that the degree of preference for $\mathrm{X}$ is the brain's estimate of the potential benefit of trying to get $\mathrm{X}$, therefore depending both on the probability that the act will be successful, and the value and cost of success and failure, respectively. In the form of a simple equation, our central theoretical assumption is that

Degree of preference for $\mathrm{X}=$ the expected utility of acting to obtain $\mathrm{X}$

$$
=P(\operatorname{getX}) \times V(\operatorname{get} \mathrm{X})+P(\neg \operatorname{get} \mathrm{X}) \times V(\neg \operatorname{getX}),
$$

where ' $\mathrm{get} X$ ' is the proposition that the observer's act to obtain $\mathrm{X}$ is successful, $P$ is probability, $V$ is estimated utility, and $\neg$ is negation. In the real world the cost of trying but failing to obtain $\mathrm{X}$ may vary depending on the nature of $\mathrm{X}$. However, for the experimental effects we analyze here in light of the above hypothesis, there tends to be 
little or no reason to believe that this cost of failure varies across stimuli, and thus equation (1) will be driven by the first product, namely that

Degree of preference for $\mathrm{X} \approx P(\operatorname{get} \mathrm{X}) \times V(\operatorname{get} \mathrm{X})$.

Also, we will assume that the main utility of obtaining $\mathrm{X}$ is simply the utility of having $\mathrm{X}$, rather than, say, some intrinsic reward in having gone through the act of obtaining $\mathrm{X}$. It follows that we may write

Degree of preference for $\mathrm{X} \approx P(\operatorname{get} \mathrm{X}) \times V(\mathrm{X})$.

That is, degree of preference for an object $\mathrm{X}$ depends on the utility of $\mathrm{X}$, which is hardly surprising, but also on the probability that the observer can actually obtain $\mathrm{X}$. Although outside the scope of this paper, we note that this fits well with endowment effects where people prefer an object already in their possession over that same object type not yet in their possession (Thaler 1980; Kahneman et al 1991), for, although the utility of the objects are identical in each scenario, the probability of obtaining $\mathrm{X}$ is greater when in hand than when not.

For the remainder of this paper we discuss how visual exposure is expected to modulate degrees of preference by modulating one or both terms of equation (2); that is, by either changing the estimated probability of obtaining $\mathrm{X}$ (section 2), which explains the initial preference for familiarity; or by changing the estimated utility of $\mathrm{X}$ (section 3), which explains the eventual preference for novelty. We will also describe (section 4) how in light of our functional hypothesis it is not mysterious that affect can be modulated subconsciously, ie via stimuli the observer does not consciously recognize having seen. In all our forthcoming discussions we assume that the visual stimuli in exposure-related experiments are treated as potential objects, as opposed to simpler features.

\section{Exposure to $X$ enhances preference for $X$ by increasing the estimated probability of obtaining $\mathrm{X}$}

Let us begin by considering an observer who is visually exposed one or several times to an object of type X. First let us ask how the estimated probability of obtaining X, $P($ get $\mathrm{X})$, changes. In most scenarios it will increase because visual exposures of $\mathrm{X}$ provide evidence that objects of type $\mathrm{X}$ are in the vicinity, potentially ready for the taking. There are unusual circumstances in which more instances of $\mathrm{X}$ can warrant lowering the probability of obtaining X. For example, imagine a kind of bird only typically seen as it bursts out from the cover of grass in a meadow. Each visual exposure to a bird of this type is, then, one less bird nearby for the taking. Typically, however, visual exposure to an object of type $\mathrm{X}$ does not entail the inability to acquire $\mathrm{X}$, and will therefore enhance the probability of obtaining it. And, importantly, there is no reason to believe that the stimuli used in 'exposure' experiments possess cues that they have properties like in the bird scenario.

Now let us consider how a small number of visual exposures might modulate the observer's estimated utility of possessing an object of type $\mathrm{X}$, or $V(\mathrm{X})$. New evidence acquired by an observer can lead him to modulate his estimate of the judged utility. For example, perhaps an observer initially believes (not necessarily consciously) that some insect grub has a 0.9 probability of being a good meal with a value of 5 , but a 0.1 probability of being poisonous with a value of -20 . The estimated utility is $V(\mathrm{X})=(0.9)(5)+(0.1)(-20)=2.5$. But if the observer sees, say, a chimpanzee eat one, this may serve to enhance the probability that the grub is a good meal to 0.95 , thereby raising the grub's expected utility to $(0.95)(5)+(0.05)(-20)=3.75$. More generally, there may be a continuum of possible utilities for the grub with a subjective probability distribution over it, a distribution that can change as evidence dictates. 
Specific learning experiences like the grub example above can serve to modulate the expected utility of objects, but for our purposes here we are interested in whether exposure itself - and not the properties that an observer can glean from the stimuluscan lead to rational modulations of judged utility. One can concoct contrived examples where exposure could reduce the estimated utility: eg imagine two kinds of fly, where the tasty ones fly through your locale only once per day, and the foul-tasting flies come back multiple times. There should be a large drop in judged utility at the second exposure. Outside of bizarre scenarios like this, however, several exposures would not appear able to rationally much lower the estimated utility of an object. There are also situations where the estimated utility might increase at first: eg after seeing an object of type $\mathrm{X}$ and suffering no negative consequences by being in its proximity, the observer's subjective estimate of the utility of X can rise. Such 'neophobia' has, in fact, been the primary hypothesis for the functional advantage of the mere exposure effect (eg Zajonc 1998; Monahan et al 2000). Although it is plausible that the estimated utility of X may at first increase in some cases for neophobic reasons, many classes of novel stimuli would seem to be intrinsically unthreatening-we are not initially fearful of every new thing we see-especially the kinds of stimuli used in exposure-related experiments. Neophobia is, at any rate, an insufficiently rich foundation to explain the set of exposure-related phenomena discussed in this paper, and summarized in table 1 (right column). In this light, we will assume here that the estimated utility of $\mathrm{X}$ does not tend to change much upon seeing a small number of visual exposures (although recognizing that in some cases neophobia could drive an initial increase). In the following section, however, we will suggest that very many exposures can justify a change in the rational estimated utility.

In sum, then, several visual exposures to an object of type $\mathrm{X}$ will typically raise the probability of acquiring the object if you were to try to obtain it, but will not modulate the estimated utility of possessing the object. Typically, then, a small number of visual exposures will tend to enhance preference for objects of type X. We speculate that this simple fact underlies fundamental exposure effects, where visual exposure to X initially tends to enhance preference (Zajonc 1968; Zajonc et al 1972; Bornstein 1989; Shimojo et al 2003) - see table 1, A.1.

Although exposure effects have been shown for both passive exposure (Zajonc 1968; Zajonc et al 1972; Bornstein 1989) and active exposure where the observer can choose on which object to fixate (Shimojo et al 2003), our hypothesis predicts certain differences between passive and active exposure. Because people have a tendency to look at what they prefer-something we are not explaining here, but something more obviously functionally advantageous - in active (but not passive) exposure scenarios there will be a positive feedback between seeing and preference. Seeing enhances liking, liking leads to more seeing, and so on. If we assume that a decision is taken when the relative preference for one object over the other reaches some threshold, then we expect that when preference decisions are made where the observer can freely actively gaze, there should be an avalanche of gazing at the to-be-chosen object just before it is chosen. Such an avalanche would not, however, be expected for non-preference decisions (eg "which object is more round?") because non-preference decisions will not typically be affected by the frequency of exposure, whereas the probability of obtaining an object (and thus preference in our view) does depend on this. Indeed, such an avalanche of fixations on the to-be-chosen object just before the decision-which we call the 'cascade effect' - has been found for preference decisions, but not for nonpreference decisions (Shimojo et al 2003) - see table 1, A.2.

Our view of preference makes a further prediction concerning this avalanche of gazing during a preference decision. Suppose the two objects from which the observer must choose differ considerably in their initial preference. In this case little or no positive 
Table 1. Effect of visual exposure on preference for X.

Key property of our Prediction of our hypothesis hypothesis for prediction

Empirical effect

Exposure effect (Zajonc 1968; Zajonc et al 1972; Bornstein 1989; Shimojo et al 2003)

Cascade effect (Shimojo et al 2003)

Enhanced cascade effect for initially similarly-preferred objects (Shimojo et al 2003)

(B) The estimated utility of the object, $V(\mathrm{X})$

(B.1) Overexposure to object type $\mathrm{X}$ eventually serves as evidence that $\mathrm{X}$ is overabundant, inconsistent with it having a high utility, and thereby lowering the estimated utility. The expected utility of acting to obtain $X$ eventually is dampened, and therefore the degree of preference for $\mathrm{X}$ should eventually dampen [either counteracting or reversing the initial preference increase in (A.1)].

(B.2) Because evidence that an object of type $\mathrm{X}$ is potentially obtainable nearby can occur with few exposures but evidence that $\mathrm{X}$ is overabundant requires very many exposures, the time-scale for the eventual lowering of the utility of $\mathrm{X}$ is long compared to that of the initial raising of the probability of obtaining $\mathrm{X}$. Therefore, the degree of preference should initially rise relatively quickly with exposure, and only later, and more slowly, attenuate.

(B.3) Massed visual exposure to an object of type X (eg an exposure schedule that is more homogeneous or has shorter interstimulus delays between presentations of X) tends to accelerate the accumulation of evidence of overabundance, thereby devaluing $\mathrm{X}$ earlier, and dampening the exposure effect.

(B.4) Simpler stimuli are a priori more common, and fewer exposures are required before evidence of overabundance is achieved. Devaluation of $\mathrm{X}$ occurs more quickly, thereby dampening the exposure effect.

(C) The expected utility of acting to obtain $\mathrm{X}$ is affected by some variables that do not require conscious recognition (exposure schedule information)

(C.1) The effects above rely only on exposure schedule information, not on conscious judgments about the objects, and thus conscious recognition of the objects is not necessary.

(C.2) In fact, the effects above are just one potential contributor to preference change, and conscious judgments are another. When conscious recognition also contributes, the predicted dynamics in (A) and (B) above due simply to the exposure schedule will be dampened. Accordingly, when visual exposure occurs without conscious recognition, the exposure effect should be enhanced.
Inverted "U" (Berlyne 1970; Zajonc et a 1972; Kail and Freeman 1973; Stang and O'Connell 1974; Bornstein 1989)

Quick rise, slow fall (Stang and O'Connell 1974; Bornstein 1989)

Massed versus spaced exposure schedule (Berlyne 1966, 1970, 1971; Kail and Freeman 1973; Stang and O'Connell 1974; Bornstein 1989)

Simple versus complex stimuli (Berlyne 1970, 1971; Bornstein 1989)

Without conscious recognition (Moreland and Zajonc 1977; Kunst-Wilson and Zajonc 1980; Bornstein 1992; Johansson et al 2005)

Stronger preference increase without conscious recognition (Bornstein 1989, 1992; Bornstein and D'Agostino 1992) 
feedback is expected prior to a decision. For example, if the initial preference for one object relative to the other is already above the threshold needed for taking a decision, then the decision can be taken without any positive feedback occurring, and thus there is no cascade effect. If, instead, the initial preferences differ considerably but not above the threshold required for taking a decision, then the positive feedback will begin to occur, but will only proceed for a short time before threshold is achieved, and so the cascade effect will be weak. However, now let us suppose that the two objects from which the observer must choose initially have approximately the same degree of preference. Because of the positive feedback between visual exposures and preference, any small asymmetry that occurs between the objects - ie one of the objects begins to be preferred over the other-will tend to become amplified. Because the initial preferences were approximately matched, a large avalanche of gazing will occur on the to-be-chosen object before the threshold is overcome for a decision, and a large cascade effect is therefore expected. Therefore, our hypothesis predicts that the cascade effect should be stronger when the observer must make a preference decision between objects initially more similarly preferred. This is, in fact, what experiments have shown (Shimojo et al 2003), providing further support for our view of preference put forth here (see table 1, A.3).

In sum, visual exposure to an object $X$ tends to initially raise the probability that one can obtain that object if one were to try, thereby raising the expected utility of acting to obtain $\mathrm{X}$. This may explain why visual exposure, whether passive or active, tends to initially enhance preference. In natural situations where an observer can actively fixate on the objects from which he may choose, there is a positive feedback between seeing and liking which can serve to drive a preference decision when the initial degrees of preference are similar. We suggest that this may be the functional foundation of the cascade effect-see table 1, A.

\section{Overexposure to $\mathrm{X}$ lowers preference for $\mathrm{X}$ by decreasing the estimated utility of $\mathrm{X}$}

In the previous section we suggested that the early positive effects of visual exposure on degree of preference may be due to an increase in the estimated probability that the observer can obtain the seen objects. The other term driving degree of preference in equation (2) - the estimated utility of an object, $V(\mathrm{X})$ - did not play a role. The estimated utility is not, however, immune to change. Although several visual exposures to an object of type $\mathrm{X}$ are not in themselves evidence that could much affect the estimated utility of $\mathrm{X}$, there is a simple reason why sufficiently many exposures eventually can do so: it provides increasing evidence that objects of type $\mathrm{X}$ are 'overly common', and overly common objects are, in turn, probably not valuable. Said another way, valuable objects tend to be rare because other individuals typically recognize their utility and acquire them, and so overabundance is evidence of low utility. (The converse is, by the way, not true, for many or most rare things are not valuable.) For example, suppose an observer initially gives a probability of 0.8 that objects of type $\mathrm{X}$ have a high utility of 10 and are accordingly rare, and a probability 0.2 that objects of type $\mathrm{X}$ are worthless. The estimated utility of $\mathrm{X}$ begins, then, at 8 . After being exposed very many times to objects of type $\mathrm{X}$, however, the evidence becomes steadily more inconsistent with $\mathrm{X}$ being rare, and thus the probability that it is rare, and of high utility 10 , may fall from 0.8 to, say, 0.1 , and so the estimated utility falls from 8 down to 1 . Thus, the estimated utility will tend to fall as evidence accumulates that objects of type $\mathrm{X}$ are overabundant. Evidence of overabundance will inherently require more exposures than evidence that an object is nearby and obtainable; this is because the former requires that one's estimate of the average global abundance (ie across locales) be raised, and the prior on this will tend to be tight (ie less uncertainty), whereas the prior on one's ability to obtain an object in one's locale will be wide (ie more uncertainty). 
(Intuitively, we are more sure that coins on average land on heads with probability $1 / 2$ than we are that some particular coin lands on heads with probability $1 / 2$.) A consequence is that the decrease in estimated utility will occur after many exposures, whereas the rise in the probability of obtaining the object will occur immediately (after only a few exposures).

Thus, after very many exposures - or at overabundance - the probability of obtaining $\mathrm{X}$ is approximately constant and the estimated utility of possessing $\mathrm{X}$ begins to fall (ie the subjective probability distribution over the possible utilities of possessing the object shifts downward). In light of our view of preference, then, we are led to the prediction that, although visual exposure can initially raise an observer's degree of preference as discussed in the previous section, a large number of visual exposures can eventually provide evidence of overabundance which, in turn, is evidence that the object type is of low utility, thereby lowering the degree of preference for this object type. That is, one expects an inverted $\mathrm{U}$, with a fairly quick rise, and a later eventual fall that occurs more slowly, which is consistent with what in fact occurs (Berlyne 1970; Zajonc et al 1972; Kail and Freeman 1973; Stang and O'Connell 1974; Bornstein 1989). In our view, then, the inverted $U$ is comprehensible: as a function of the number of exposures, the degree of preference for $\mathrm{X}$ may follow an inverted $\mathrm{U}$ because the expected utility of acting to obtain $\mathrm{X}$ follows an inverted $\mathrm{U}-$ see table 1, B.1 and B.2; and figure 1. Our hypothesis concerning the eventual downturn in preference is not necessarily inconsistent with the idea that it is due to 'boredom' (Berlyne 1970), because boredom is put forth as a kind of mechanism, and we are here trying to explain why such a mechanism would be advantageous. (Alternatively, at times 'boredom' seems to be used more as a phenomenological description of the downturn effect, in which case there is still no inconsistency with our account.)

Our hypothesis makes some further predictions in this regard. Notice that evidence of overabundance is the primary contributor to lowering the degree of preference over many exposures. That is, eventually this factor starts pushing the degree of preference back down after the initial increase discussed in section 2. This counterweight to the initial preference enhancement can be accelerated by accelerating the rate at which information accumulates that suggests that the object type is overly abundant. Any such acceleration would serve to dampen the strength of the rise in the exposure effect, either slowing it down or reversing it. One obvious way to accelerate the accumulation of evidence of overabundance is by making the exposure schedule to object type $\mathrm{X}$ more homogeneous, ie with fewer other object types visually exposed in between presentations of $\mathrm{X}$. A related way to do this would be to decrease the interstimulus delay between exposures of $\mathrm{X}$. Each of these is therefore expected to dampen the strength of the rising degree-of-preference feature of the exposure effect. And, indeed, as predicted, these have long been noted to dampen the exposure effect (Berlyne 1966, 1970, 1971; Kail and Freeman 1973; Stang and O'Connell 1974; Bornstein 1989) - see table 1, B.3.

Another way to accelerate the rate at which evidence accumulates for overabundance is to have an object type that starts with a higher a priori estimate for its abundance, for then fewer exposures will be required before evidence is strong for overabundance. For example, all things equal, simpler objects tend to have greater a priori probability of being common in the world. One of the most fundamental reasons for this is that simpler stimuli can occur alone or as constituents in a large number of more complex stimuli, but not vice versa. A second reason concerns recent research into the natural scene probabilities of shapes showing that simpler shapes (which correlates highly with having fewer angles-Hochberg and McAlister 1953; Attneave 1957; Arnoult 1960) tend to be the more ecologically probable (Changizi et al 2006), suggesting that judged visual complexity may be driven by ecological improbability. 


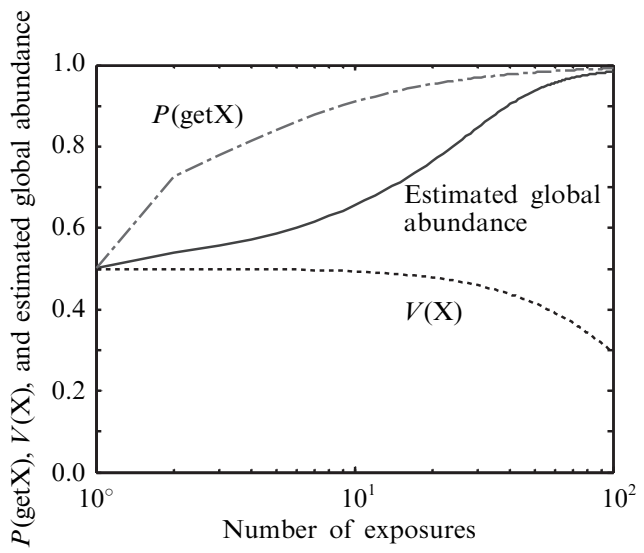

(a)

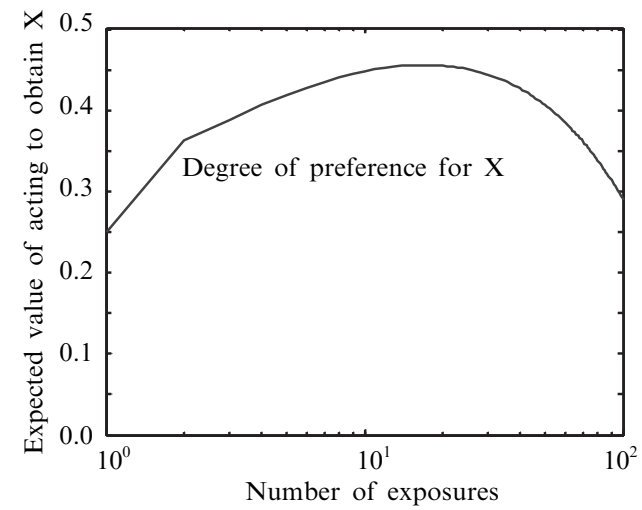

(b)

Figure 1. (a) Illustration of the general manner in which the key variables hypothesized to affect degree of preference vary with the number of exposures, and (b) how degree of preference (which our theory identifies with the expected utility of acting to obtain X) consequently varies. Notice that the $x$-axis is logarithmic. One can see that after several visual exposures the probability of obtaining $\mathrm{X}, P($ getX), quickly rises, for it provides evidence that there are potentially available $\mathrm{Xs}$ nearby. The expected utility of acting to obtain $\mathrm{X}$ accordingly rises [the product of $\mathrm{P}($ getX) and $V(\mathrm{X})]$, as shown in (b). The estimated utility of $\mathrm{X}, V(\mathrm{X})$, does not begin to change until sufficiently many exposures have occurred, and evidence consequently accumulates that $\mathrm{X}$ may be overabundant, as shown by the relatively slowly increasing estimated global abundance. Only once this global abundance is high - or it reaches 'overabundance'-does it begin to provide evidence that $V(\mathrm{X})$ may be lower than originally estimated. As $V(\mathrm{X})$ begins to fall, it serves as a counterweight to the increasing $P(\mathrm{getX})$, slowing down the increase in preference, and eventually lowering preference. [The curves shown here are meant only to indicate the general qualitative relationships our theory predicts. The $P($ getX) and global-abundance curves shown here are two examples of how the estimated frequency of red balls in an infinite urn vary upon pulling out more and more red balls, but where the former curve has a wide prior probability distribution over the possible urn frequencies, and the latter a narrow distribution (capturing that it takes a lot of evidence before one is willing to modulate one's judgment about how generally abundant an object of type $\mathrm{X}$ is, rather than just in your current locale). The estimated utility, $V(\mathrm{X})$, here (arbitrarily scaled to fit on the plot) has been made to depend on the estimated global abundance so that as the global abundance rises into overabundance, it forces the Bayesian reasoner to lower the estimated utility. The important qualitative relationship between global abundance and $V(\mathrm{X})$ is that $V(\mathrm{X})$ is not much affected until the estimated global abundance becomes high.]

Fewer visual exposures therefore tend to be needed for simpler stimuli before the exposures amount to evidence that objects of that type are overabundant, and thus probably not valuable. Therefore, our hypothesis predicts that the rising preference regime of the exposure effect should be weaker for simpler stimuli. Indeed, this has been shown in experiments (Berlyne 1970, 1971; Bornstein 1989), providing further support for our view of preference-see table 1, B.4.

In sum, although visual exposure to an object $\mathrm{X}$ tends to initially raise the expected utility of acting to obtain $\mathrm{X}$ - and thus raise preference-after many exposures there is a counter-tendency due to the accumulation of evidence that $\mathrm{X}$ is overabundant, and thus probably not high utility. We speculate that this explains the adaptive value of the inverted $U$ for preference as a function of visual exposure. Consistent with this, the strength of the rising part of the exposure effect is modulated as predicted, in that accelerating the rate at which evidence accumulates for overabundance-either via a more homogeneous exposure schedule, shorter interstimulus delays, or the use of a simpler stimulus - dampens the rising regime of the exposure effect-see table 1, B. 


\section{Preference can be modulated without conscious recognition because these rational modulations to preference do not require conscious recognition}

Consciously recognizing an object of type $\mathrm{X}$ allows one to make potentially complex judgments about the expected utility of attempting to obtain $\mathrm{X}$. Complex reflective questions one might ask oneself include: "How difficult was it for my brother to acquire?" $[P($ getX) $)$, and "How much utility is an object like that likely to give me were I to obtain one?" $[V(\mathrm{X})]$. However, note that the kind of information we have discussed in the previous two sections (and summarized in table 1, A and B) can be inferred from visual exposure information alone, information that does not necessarily require having conscious recognition of the objects and carrying out subsequent complex conscious tasks. We believe this underlies one of the most surprising aspects of visual exposure effects: that they do not require conscious recognition of the objects (Moreland and Zajonc 1977; Kunst-Wilson and Zajonc 1980; Bornstein 1992). In fact, in more recent experiments this has been demonstrated in situations where observers can freely gaze and make a preference decision. Johansson et al (2005) carried out an experiment where, after observers make a preference decision, the experimenter shows each observer the unchosen object, but via a sleight of hand the unchosen object appears to come from the same location as the chosen object, and the experimenter falsely tells the observer that the unchosen object is what the observer chose. The observer is then asked what stimulus features helped her/him make the decision. Surprisingly, observers are often not aware that they are being shown the unchosen object, and they provide reasons that apply to the unchosen object; apparently, observers often do not consciously recognize their choice at all (see also Adolphs et al 2005) - see table 1, C.1.

Our hypothesis not only expects that conscious recognition is not needed in order for visual exposure to modulate degree of preference, but that conscious recognition is expected to dampen these effects. The reason for this is that the predicted changes to degree of preference that we have discussed in this paper presume that the only information being utilized to rationally modulate degree of preference is exposure schedule information. In many natural situations, observers potentially have both exposure schedule information and consciously accessible information about the object, in which case the predicted degree of preference modulations discussed in this paper would be attenuated, for they would be competing with the information from conscious recognition of the object and any subsequent judgment. Consistent with this, when visual exposure is carried out in such a way that the observer does not consciously recognise having seen it, the preference-increase effects are actually stronger than when observers have recognized the objects (Bornstein 1989, 1992; Bornstein and D'Agostino 1992). (It is currently unclear how the preference-decreasing regimes are modulated by subliminality-see table 1, C.2.)

We suggest, then, that our theory of preference explains why there appears to be a 'non-cognitive' (or perhaps better to say, 'non-conscious'), or 'hot', route to affect modulation (Moreland \& Zajonc 1977). This 'hot' mechanism exists because visual exposure information alone, without conscious judgment, has implications for the expected utility of one's actions, and the mechanism was therefore selected for it. In fact, one might speculate that for most non-human mammals conscious deliberation plays a smaller role in modulating preference than for us, and that this non-conscious mechanism relying only on visual (or sensory, more generally) exposure information may be evolutionarily more ancient - see table $1, \mathrm{C}$.

\section{Conclusion}

We emphasize that our hypothesis is a functional one, not making any claims about mechanisms. It should therefore not be thought of as a competitor to proposed mechanisms such as response competition (Harrison 1968; Harrison and Zajonc 1970), positive 
habituation and boredom (Berlyne 1970), and perceptual fluency (Bornstein and D’Agostino 1992; Reber et al 1998, 2003; Winkielman et al 2003). In fact, we believe our work provides a novel plausible interpretation for the functional advantage of perceptual fluency mechanisms: perceptual fluency and expected utility are probably highly correlated in the natural environment. Competing hypotheses to ours would be hypotheses about the adaptive advantage of exposure-related effects. There has been speculation about potential functional advantages. For example, response competition and perceptual fluency proponents have pointed to neophobia as one potential functional advantage (Zajonc 1998; Monahan et al 2000; Reber et al 2003; Winkielman et al 2003), and perceptual fluency proponents have additionally pointed to potential advantages concerning prototypicality/symmetry and cues to cognitive progress (Winkielman et al 2003). But these functional hypotheses were only 'pointed to', and no attempt has thus far been made to make and test predictions of these functional hypotheses.

In light of our functional hypotheses, seeing is useful for more than just determining what is around you. When you look in the course of normal behavior, you see objects, and the frequencies with which you see objects are not irrelevant to the expected utility of potential acts aimed at obtaining objects, and thus, in our view, not irrelevant for preference. A small number of exposures to an object of type $X$ can quickly raise the probability of obtaining an $\mathrm{X}$, raising the expected utility of acting to obtain X. Here we have speculated that this explains the functional advantage of increasing degree of preference with visual exposures (or 'familiarity preference'table 1, A. A large number of exposures to $\mathrm{X}$, however, eventually suggests that Xs are so abundant in the environment that it is unlikely they are valuable (for if they were valuable, then they would probably be rare), which eventually lowers the expected utility of an act to obtain $\mathrm{X}$ - table $1, \mathrm{~B}$ and also figure 1 . We suggest that natural selection has discovered this, and so we have been designed to unconsciously (table 1, C) keep track of exposure frequency information, and to modulate preference accordingly. That is, we suggest that evolution has instilled in us mechanisms which modulate preferences as a function of visual exposure so that the degrees of preference tend to track the expected utilities of goal-directed acts toward objects.

There are several limitations to our functional hypothesis. First, although our framework is not specifically visual and may apply to exposure-related effects in other modalities such as audition and olfaction, our work here would probably not apply well to gustatory stimuli which in natural scenarios are typically already possessed in the mouth by the observer (and exposure and reward will be more inextricably linked). Second, although the fundamental idea underlying our hypothesis for preference-that preference for $\mathrm{X}$ is driven by the expected utility of acting to obtain $\mathrm{X}$ - is simple, in real scenarios it can be very complicated to theoretically determine the numerical values of the terms in equation (1) so as to make predictions. Because of the relatively simple nature of exposure-related experiments, we were able to simplify it to equation (2), and we were able to make plausible arguments concerning how these two terms should vary with exposure. The extent to which our framework would extend to complex naturalistic settings is unclear. Third, our framework is intended only to address our non-conscious, 'hot' mode preferences, not necessarily our conscious judgments of what we prefer. Although there are connections between these, we have no special insight into how they interact.

Acknowledgments. Support for this research was given by 5F32EY015370-02, NIH (to MAC), and JST.ERATO, Japan (to SS).

\section{References}

Adolphs R, Tranel D, Koenigs M, Damasio A R, 2005 "Preferring one taste over another without recognizing either" Nature Neuroscience $\mathbf{8} 860-861$

Arnoult M D, 1960 "Prediction of perceptual responses from structural characteristics of the stimulus" Perceptual and Motor Skills $11261-268$ 
Attneave F, 1957 "Physical determinants of the judged complexity of shapes" Journal of Experimental Psychology $53221-227$

Berlyne D E, 1966 "Curiosity and exploration" Science 153 25-33

Berlyne D E, 1970 "Novelty, complexity, and hedonic value" Perception \& Psychophysics 8 279-286

Berlyne D E, 1971 Aesthetics and Psychobiology (New York: Appleton-Century-Crofts)

Bornstein R F, 1989 "Exposure and affect: Overview and meta-analysis of research, 1968 - 1987" Psychological Bulletin $106265-289$

Bornstein R F, 1992 "Subliminal mere exposure effects", in Perception without Awareness: Cognitive, Clinical, and Social Perspectives Eds R F Bornstein, T S Pittman (New York: Guilford Press) pp $191-210$

Bornstein R F, D’Agostino P R, 1992 “Stimulus recognition and the mere exposure effect” Journal of Personality and Social Psychology 63 545-562

Changizi M A, Zhang Q, Ye H, Shimojo S, 2006 "The structures of letters and symbols throughout human history are selected to match those found in objects in natural scenes" American Naturalist 167 E117-E139

Eagly A H, Chaiken S, 1993 The Psychology of Attitudes (Belmont, CA: Thompson)

Harrison A A, 1968 "Response competition, frequency, exploratory behavior, and liking" Journal of Personality and Social Psychology $9363-368$

Harrison A A, Zajonc R B, 1970 "The effect of frequency and duration of exposure on response competition and affective ratings" Journal of Psychology 75163 - 169

Hochberg J, McAlister E, 1953 "A quantitative approach to figural 'goodness" Journal of Experimental Psychology 46361 - 364

Johansson P, Hall L, Sikström S, Olsson A, 2005 "Failure to detect mismatches between intention and outcome in a simple decision task" Science 310 116-119

Kahneman D, Knetch J L, Thaler R H, 1991 "Anomalies: The endowment effect, loss aversion, and status quo bias" Journal of Economic Perspectives 5 193-206

Kail R V Jr, Freeman H R, 1973 "Sequence redundancy, rating dimensions, and the exposure effect" Memory \& Cognition $1454-458$

Kunst-Wilson W R, Zajonc R B, 1980 "Affective discrimination of stimuli that cannot be recognized" Science $207557-558$

Monahan J L, Murphy S T, Zajonc R B, 2000 "Subliminal mere exposure: specific, general, and diffuse effects" Psychological Science $11462-466$

Moreland R L, Zajonc R B, 1977 "Is stimulus recognition a necessary condition for the occurrence of exposure effects?" Journal of Personality and Social Psychology 35191 - 199

Reber R, Schwarz N, Winkielman P, 2003 "Processing fluency and aesthetic pleasure: Is beauty in the perceiver's processing experience?" Personality and Social Psychology Review $8364-382$

Reber R, Winkielman P, Schwarz N, 1998 "Effects of perceptual fluency on affective judgments" Psychological Science 9 45-48

Shimojo S, Simion C, Shimojo E, Scheier C, 2003 "Active-gaze exposure-effect both reflects and influences preference" Nature Neuroscience $61317-1322$

Stang D J, O'Connell E J, 1974 "The computer as experimenter in social psychological research" Behavior Research Methods \& Instrumentation $6223-231$

Thaler R, 1980 "Towards a positive theory of consumer choice" Journal of Economic Behavior and Organization $139-60$

Winkielman P, Schwarz N, Fazendeiro T A, Reber R, 2003 "The hedonic marking of processing fluency: Implications for evaluative judgment", in The Psychology of Evaluation: Affective Processes in Cognition and Emotion Eds J Munsch, K C Klauer (Mahwah, NJ: Lawrence Erlbaum Associates) pp $189-217$

Zajonc R B, 1968 "Attitudinal effects of mere exposure" Journal of Personality and Social Psychology, Monograph Supplement 9 1-27

Zajonc R B, 1998 "Emotions", in The Handbook of Social Psychology Eds D T Gilbert, S T Fiske, G Lindsey (Boston, MA: McGraw-Hill) pp $591-632$

Zajonc R B, Shaver P, Tavris C, Van Kreveld D, 1972 "Exposure, satiation, and stimulus discriminability" Journal of Personality and Social Psychology 21 270-280 
Conditions of use. This article may be downloaded from the E\&P website for personal research by members of subscribing organisations. This PDF may not be placed on any website (or other online distribution system) without permission of the publisher. 